\title{
"Poverty is our Biggest Enemy": Canadian Nursing Students' International Learning Experiences (ILEs)
}

\author{
By Hannah Ashwood-Smith ${ }^{*}$, Lorelei Newton ${ }^{\dagger}$ \& Renate Gibbs ${ }^{*}$
}

\begin{abstract}
International Learning Experiences (ILES) have been a cornerstone of global health education for nursing programs throughout the world. Camosun College's Nursing Department (Victoria, $B C$, Canada) has conducted numerous ILEs in many developed and developing countries for over a decade with only anecdotal evidence to support these rich yet challenging international placements. Thus, the principle objectives of this research aimed to explore the impact of study abroad placements on students' global health knowledge acquisition, and personal and professional growth, in addition to understanding the important perspectives of the host countries. The methodology combined qualitative and quantitative components and employed a global health framework. The data collection tools included focus group discussions, global health themed critical reflections, a survey, and a structured questionnaire. An interpretive description approach guided the analysis. The results revealed the complexity of the students' personal and professional journey as they incorporated global health concepts into their novice practice. Furthermore, health promotion was a critical dimension of the data illuminating student's enhanced knowledge levels of principles of upstream thinking and effective health education strategies. Cultural competence as a key learning outcome fostered complex ethical discussions supporting the concept of cultural comportment. It is hoped that these research findings, coupled with recommendations for best practice, will help inform the debate on the merits and challenges of ILEs, ensuring that vital concepts of global health knowledge and cultural competence are deeply embedded into future international nursing placements.
\end{abstract}

Keywords: Nursing students, Global health, Cultural competence, Personal and professional growth, International Learning Experiences

\section{Introduction}

Canada is a multi-cultural society embracing over 200 ethnic groups (Statistics Canada 2011). According to the National Household Survey, one in five people living in Canada is foreign-born representing $21 \%$ of the population, the highest of all the G8 countries (Statistics Canada 2011). Four percent of Canadians have an Aboriginal identity. Furthermore, the Canadian Nursing Association (CNA 2018) has adopted a new position statement promoting cultural competence stating all nurses have an obligation to strive for culturally competent care. For the purposes of this project, cultural competence is defined as "the ability of nurses to self-reflect on their own cultural values and how these impact the way they provide care" (CNA 2018). Therefore, it is increasingly important that nursing programs adapt curricula to reflect cultural minority's diverse health needs. Developing a cohort of culturally competent nursing graduates with a deeper awareness of global health principles will promote high quality nursing care for all Canadians (CNA 2018).

\footnotetext{
*Faculty, Camosun College, Canada

${ }^{\dagger}$ Faculty, Camosun College, Canada

${ }^{\ddagger}$ Faculty, Camosun College, Canada
} 
The Camosun College Nursing Department, Victoria, BC, Canada has offered international clinical field school experiences for Canadian student nurses in the Baccalaureate of Nursing (BSN) program for over a decade. No formal evaluation has been performed, but anecdotal evidence and student testimonials have reflected positively on these international learning experiences (ILEs). To this end, we embarked on a project to examine the impact of ILEs on students and host country institutions.

\section{Literature Review}

A number of studies have published the merits of International Learning Experiences (ILEs) or exchange programs for nursing students (Button et al. 2005, Frisch 1990, Greatrex-White 2007, Kulbok et al. 2012, Zorn et al. 1995) highlighting the importance of these unique opportunities to enhance nursing students' personal and professional growth (Greatrex-White 2007, Lee 2004). These invaluable international placements provide prospects to develop cultural competence (e.g., Campinha-Bacote 2011, DeSalles Turner 2007, Kokko 2011, Ruddock and Campesino 2002). Numerous studies report that healthcare students express a desire to gain deeper knowledge of other cultures and wish to experience a different health care system (Bohman and Borglin 2014, Burgess et al. 2014, Gower et al. 2016, Holmes et al. 2012, Kelleher et al. 2016, Kent-Wilkinson et al. 2015, Keogh and Russel-Roberts 2009, Owen et al. 2013, Wehbi 2009). Students would like a chance to improve upon their nursing knowledge (Bohman and Borglin 2014, Holmes et al. 2012, Gower et al. 2016, Kumwenda et al. 2014), learning from low-income countries, and expressed a craving to contribute towards health issues in order to make a palpable difference (Burgess et al. 2014, Gower et al. 2016, Kumwenda et al. 2014, Reimer-Kirkham et al. 2009, Wehbi 2009).

Recent literature reviews explore factors in North America and Europe which influence healthcare students' decisions to pursue ILEs (Brown et al. 2016, Kent-Wilkinson et al. 2015). Findings suggest that prior travel opportunities and volunteer experiences, coupled with family support, fuelled commitment for some students to participate in studies abroad (Burgess et al. 2014, Gower et al. 2016, Kent-Wilkinson et al. 2015, Keogh and RusselRoberts 2009, Owen et al. 2013). Challenges for students are identified as inadequate funds, potential lost wages and family considerations (Bohman and Borglin 2014, Kelleher et al. 2016, Kent-Wilkinson et al. 2015, Morgan 2012). One survey indicated that $54 \%$ of Canadian nursing schools incorporate international placements into their curricula (DeLong 2015) speaking to the need for well planned ILEs which truly enhance students' evolution. Financial barriers seem to be the overarching deterrent for nursing schools and/or students considering international placement options (Kelleher et al. 2016).

Global literature reveals a dearth of data exploring the host country's perceptions of these ILEs (DeLong 2015, Underwood et al. 2016). Furthermore, students' knowledge acquisition on core global health concepts have rarely been studied in detail, although past findings support the notion 
that ILEs can have a positive influence on students' cognitive development (Button et al. 2005, Zorn et al. 1995, Frisch 1990). There have been some concerns raised by faculty that students returning from these ILEs are inadequately prepared clinically for subsequent placements, lacking certain necessary psychomotor skills based on Canadian nursing standards.

\section{Methodology}

\section{Ethics}

Ethical approval for the study was obtained in writing from the Camosun College Research Ethics Board; New Zealand and the Philippines institutions were approached for written consent to conduct the study within their hospital and community settings. Host country questionnaires were distributed and collected through a confidential process each week. Written informed consent was acquired from all Canadian nursing students and verbal informed consent was obtained from host country staff nurses prior to administration of confidential questionnaires. Data was stored in secure files with names removed from the critical reflections prior to data analysis. Focus group discussions were conducted by a faculty member who did not accompany students and had no involvement with any student evaluations. Informed consent was obtained from the nursing students for each focus group (pre and post departure).

Design

A combination of qualitative and quantitative data collection techniques were employed using an adapted version of Riner's (2011) framework. Focus group discussions (FGDs) $(\mathrm{n}=4)$, global health themed critical reflections (CRs) $(n=32)$, surveys $(n=12)$ and a structured questionnaire $(n=62)$ were used to address four study objectives examining host countries perspectives of the ILEs, and the Canadian nursing students' motivating factors, and personal and professional growth, with a strong emphasis on global health concepts.

\section{Sample}

Student participants ranged in age from 20 to 34 years old with mean age of 24. There were 22 students in total; 16 went to the ILE in the Philippines (seven first year and nine second year students) and six students attended the New Zealand ILE (all second-year nursing students). All nursing students consented to participate in at least one aspect of the research project.

Host country nurses $(n=62)$ took part and were purposively selected after working with Camosun College nursing students; 41 responded to the questionnaire from the Philippines and 21 from New Zealand. $70 \%$ of the host country nurses surveyed were unit staff with $13 \%$ nurse educators, $9 \%$ senior nurse managers, $7 \%$ outpost nurses and $1 \%$ other. 


\section{Study Setting}

\section{The Philippines}

The Filipino ILE was located centrally in a large local hospital which served impoverished populations with limited medical coverage. Camosun College nursing students learned to provide direct support and advocacy across the life span while caring for patients on a number of units. This setting provided all levels of care including male and female surgery, operating rooms, general medicine, pediatric and adult emergency services as well as pediatric and obstetrical in-patient services. A smaller neurological stroke unit, and pediatric, neonatal and adult intensive care units were also selected to assist students in meeting their learning outcomes.

\section{New Zealand}

The New Zealand ILE was located on the North Island of New Zealand. Camosun College nursing students learned about divergent health systems working in several settings: a large tertiary referral hospital, Whanau Care Services and Pacific Support Services. These services link up with individual patients and their families to provide direct support and advocacy for the Maori and Pacific Island populations. Community experiences gave students an opportunity to work under supervision in the Ora Toa Health Services. Reflecting the cultural diversity of New Zealand, this range of sites and services provided unique learning experiences for students to care for patients with poorer health outcomes than the general population.

\section{$\underline{\text { Student Preparation }}$}

All students were required to attend a full day cultural workshop hosted by Camosun's International department prior to departure. Additionally, several orientation sessions were planned by educators focusing on each country's political climate, unique health systems, and priority diseases with a specific focus on basic treatments and nursing care not previously covered by their curriculum. A simulated learning experience was conducted, ensuring students could perform comprehensive neonatal and maternal assessments. Several health promotion activities were designed for the group going to the Philippines, covering pediatric dehydration and dengue fever, including patient assessment, treatments, preventative aspects, and how to teach families the importance of, and ingredients for, homemade oral rehydration therapy. A simulated learning experience was arranged during the first week of the practicum for the New Zealand student group. This included patient assessments, clinical decision-making and the documentation expected in the host healthcare facilities. 


\section{Research Objectives}

Four research objectives were explored as follows:

1. What are the host country participant's perceptions regarding the benefits and challenges of the ILE?

2. What are the key motivating factors for students pursuing the ILE?

3. How do ILEs impact students' global health knowledge acquisition?

4. What is the impact of the ILE on students' personal and professional growth?

Although the study involved a wider focus, this paper addresses the outcomes related to the unique host country's perspectives and the benefits and challenges experienced by students in the context of global health knowledge acquisition. Their personal and professional growth is explored which inevitably covers their complex and often challenging journey towards cultural competence.

\section{Data Collection Techniques}

Multiple methods were employed to answer the study objectives which included quantitative instruments: a structured questionnaire for host country nurses and an on-line survey for nursing students. Qualitative instruments included focus group discussions and themed critical reflections for nursing students. Data was collected before and after the ILE.

\section{Questionnaire}

A structured questionnaire was designed and administered to respondent host country nursing staff $(n=62)$ working with the Canadian nursing students. Respondents ranked their satisfaction levels working with the students as per the following categories: Very Successful/Quite successful/Average/ Unsuccessful/Very unsuccessful/Unable to comment. Closed questions regarding factors that contributed positively and negatively to their experience were employed, in addition to several open-ended questions: "What has been the biggest lesson learned from hosting the Canadian nursing students?" and "How can we improve this experience from your perspective?". Data was entered into Microsoft Excel and Fluid Survey for analysis.

\section{Survey}

A survey was delivered on-line to all nursing students three months after completion of their practicum with a core focus on elements of Riner's (2011) framework (Table 1) designed to assess their global health knowledge acquisition. Respondents were asked to self-rate their competency levels on a series of topics: teaching and learning, cultural awareness, patient advocacy, and critical thinking.

The nursing students' self-rated competencies regarding cultural awareness, psychomotor skills and global health knowledge acquisition were 
obtained via an electronic survey, pre and post departure focus group discussions and themed critical reflections (with a minimum of two per student) during their five-week practicum abroad.

Table 1. Riner's (2011) Adapted Global Health Framework

Applies principles of public health to populations

Implements principles of teaching and learning that address the health of

populations at risk

Gains deeper understanding of tropical/infectious disease processes

Develops cultural competencies for working with underserved populations

Identifies cultural differences in the provision of health care resources

Provides culturally competent/sensitive health care

\section{Focus group discussions (FGDs)}

Four FGDs were held with the nursing students: two prior to their departure and two three months after their return to Canada. Topic guides were developed with an initial focus on motivating factors for choosing the ILE, foundational public health knowledge, level of preparedness for the unique experience, and the student's expectations for personal and professional growth. Sample questions from the pre FGD topic guide include: "What do you think will be your biggest challenge?" and "How do you think culture influences your practice?".

The post FGD topic guide differed slightly as the aim was to ascertain global health knowledge acquisition with a specific focus on the social determinants of health (SDOH), in addition to ethical dimensions for studying abroad. Sample questions from the post FGD topic guide included: "How did you apply the principles of public health to an at-risk population?" and "Can you identify any upstream public health strategies?". In addition, students were asked to "Describe any ethical dilemmas or situations you encountered" and "Are there any cultural considerations that will enhance your Canadian practice?".

\section{Critical reflections (CRs)}

Some studies have emphasized the importance of CR as a means of enhancing nursing students' cultural awareness (Riner 2011, Torsvik and Hedlund 2008, Ruddock and DeSalles Turner 2007), helping them to synthesize different aspects of nursing care and ensuring greater depth to their learning (Keogh and Russel-Roberts 2009).

Students completed a minimum of two themed CRs three weeks apart during the ILE experience $(n=32)$. The themes for the CRs were structured around the eleven SDOH which, loosely defined are factors that influence population/community health. These include: income, employment, education, healthy child development, physical environments, coping skills, access to health services, healthy behaviors, biology/genetics, gender and culture (Government of Canada 2018). In addition, students were prompted to consider the impact of infectious or communicable diseases on their patient's health status; however, most students selected a lens of culture, poverty, or resource allocation to reflect upon their clinical experience. 
Analysis

Quantitative data was collected through a Fluid Survey (FS) platform and analysed using descriptive statistics to ascertain measures of frequency, central tendency and variation. Analysis was completed by the research team. Qualitative data analysis was guided by an interpretive description approach (Thorne 2016). Interpretive description is a method that addresses the complexity arising out of nursing practice research and supports the excavation of subsequent convoluted and diverse data. Analysis was multi-layered with author generated groupings of broad themes that emerged from our individual readings of the qualitative data. We further coded and refined the themes for discussion. The main emerging themes are presented below. Triangulation of methodology as listed above helped to ensure more validity and rigor to the study findings, which augmented the credibility of the study.

\section{Results}

Rich data emerged from this study revealing the value of including host country staff more intentionally in the initial study design. Important incountry feedback from nursing staff was distinctly positive but also served to highlight several disparities related to language and cultural barriers, and the divergent scope of nursing practice. The Canadian students' data demonstrated a deeper understanding of the SDOH and their impact on vulnerable population's morbidity and mortality. Cultural comportment and the ethics of nursing abroad were identified as dominant themes with poverty, inequity of healthcare resource allocation, and cultural differences recognized as multifactorial issues. Health promotion was a critical dimension revealing students' enhanced knowledge levels regarding upstream thinking and effective health education strategies; specifically related to dengue fever, diarrheal disease, lung disease (asthma, pediatric community acquired pneumonia, tuberculosis), cardiac disease and diabetes. The results are presented according to the four study objectives as follows:

\section{Objective 1: Host Country's Perceptions}

The majority of health personnel from both countries (68\%) stated that the ILE was "very successful"; while $32 \%$ felt it was "quite successful". Figure 1 summarizes the benefits host staff experienced. Generally, they were impressed with the Canadian students' "humility, willingness to adapt to new situations, patience, hard work, keen assessment skills and kindness". 
Figure 1. Host Country Questionnaire Results

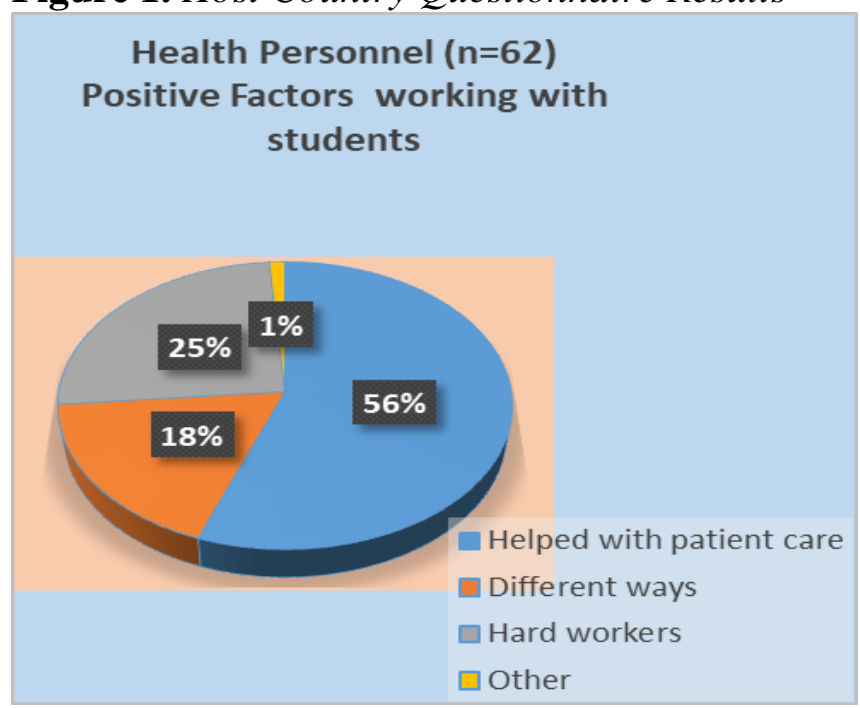

Many staff on the pediatric units were pleased with the students' willingness to engage in hands-on care and their ability to embrace their role as teachers for vulnerable families. One nurse in charge of a general pediatric ward explained:

"They have helped a lot in the pediatric general ward, taking vital signs, monitoring patients, and the teaching and learning classes on oral rehydration were very informative!".

When asked specifically about factors that impeded this ILE, although $75 \%$ of staff surveyed had no negative factors to convey, $9 \%$ stated that communication and language barriers (in the Philippines) were the most significant challenges and this, coupled with a relatively short placement, impacted students' ability to optimize learning.

"They make contributions however there are limits in labor and delivery as responses should be quick thus putting more hindrance on what students can do as they are still in orientation phase".

The different scope of practice was mentioned by numerous ward staff:

"We are hesitant to initiate certain things because this might not be practised in your country".

Students from both countries also commented on the different scope of the nurse and how this affected their ability to perform nursing care. In the Philippines there was one nurse responsible for obtaining vital signs, and another nurse solely responsible for medication administration. Canadian students were not allowed to give medications due to the difference in medication policies and procedures. In New Zealand, second-year student nurses also noted a difference between the training programs and length of training. Groups in both countries found these adjustments in practice eye 
opening. Most students were impressed with the ingenuity of the host country staff as illustrated by the following quotations:

"Filipino nurses have mastered providing care with next to no resources". (CR)

and

"Sometimes as a nurse it is the littlest things that can make the biggest impact". (CR)

Other students struggled with the roles and relationships they encountered:

"Nurses here don't question the doctors" (CR)

with another student writing:

"Doctors were very understanding, but their scope of practice is very different... strange things are done, intubation when they don't need to intubate". (CR)

\section{Objective 2: Students' Motivating Factors}

Students identified a multitude of factors that both motivated their involvement in the ILE and challenged them to move beyond their comfort zone personally and professionally as caregivers. Figure 2 and Table 2 outline a summary of the key findings.

Figure 2. Summary of Student Results

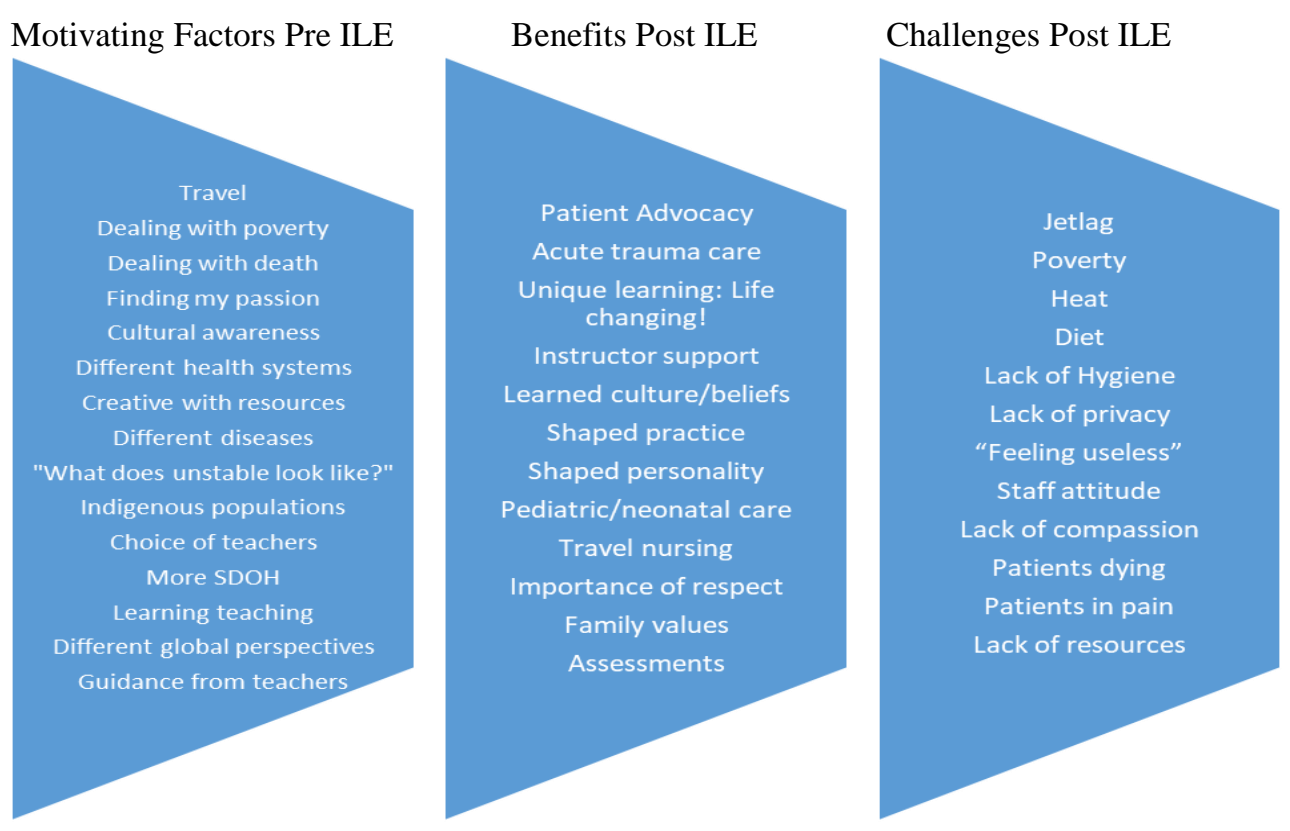




\section{Students' challenges}

As outlined above, a central concern for students attending ILEs is inadequate funds (Kent-Wilkinson et al. 2015). For this ILE experience, a greater number of students $(n=16)$ participated in the lower cost ILE in the Philippines, compared to New Zealand $(n=6)$. Although the cost played a role in their selection, an equally important consideration for many students was the opportunity to work in a developing country rather than a developed country, which would provide them with a different world view. The majority of students applied for scholarships or bursary funds to help support their programme. During both ILEs, several students discussed multiple family considerations as a part of their learning context; however, this was not commented on in the Pre or Post FGDs.

Table 2. Students' Motivational Factors

"Cultural competence, the biggest thing I can be is be open minded and not judgmental".

"I wanted to be able to experience a different culture. Everyone looks at the world differently, culture affects the way you see the world".

"In no way did I think I was going to save the world, I walked into this, I said this is my time, I wanted to experience travel nursing, but not be fully responsible for it all. I'm going to do this, and with instructors".

"I went for an opportunity to travel, I wanted the culture shock. I'm so caged and protected. I was ready to see anything that can happen, even the condition of the hospital, seeing how they function in the best way - how they function with lack of resources".

"We did priority assessments and head to toe assessments...we practised it and learned it so well and it's stayed with me".

"People there are acutely ill, there are so many things we could do-there is such a need".

Students also made several statements regarding concerns for personal safety, stress and reactions to divergent cultures. For example,

"I found that it was taxing to be aware and alert all the time...that things could have gone the other way. At times we had to advocate ourselves". (FGD)

Some students expressed concerns regarding language, communication and cultural barriers which impacted their ability to perform nursing care. Students who learned a few basic words in the local language felt this helped break down communication barriers and eased the rapport with their patients and host nurses. Instructor observations concurred with the student findings, noting enhanced relationships with those students who took the initiative to learn simple words in Tagalog or observe Pacific Island customs.

Students reported feeling upset by the extreme poverty, the profound changes in their own diet, and identified many personal challenges, namely lack of privacy and basic comforts related to their crowded living conditions. In some cases, students expressed "feeling useless" and felt confused by their perception of a "lack of compassion" demonstrated by some host nurses 
during nursing care of dying patients. Due to these complex factors, students expressed the need for a richer, more in-depth and culturally appropriate preparation. As one student explained:

"I would have liked a lot more context - this is what would have been more helpful". (FGD)

Some of the more seasoned students with travel experience benefited from their own initiative to enhance their overall preparation:

"I did a lot of research about the country ahead of time... always be aware of what is around you, don't be naïve about that". (FGD)

\section{Objective 3: Students' Global Health Knowledge}

Riner's (2011) adapted framework was incorporated to ascertain global health knowledge acquisition. Pre-departure few students could identify one $\mathrm{SDOH}$, in contrast in-country CRs revealed a much richer knowledge with most students freely journaling about witnessing ten out of $11 \mathrm{SDOH}$ including poverty, lack of hygiene, social supports, literacy, income, political/social climates, resource allocation, culture, overcrowding and unjust access to care. They witnessed SDOH in action every day.

"Be prepared for the poverty. I was prepared for poverty but not for that type of poverty!". (FGD)

In comparison, anecdotal evidence suggests that very few students who did not participate in the ILE (that is, those who stayed in Canada) discussed $\mathrm{SDOH}$ in their critical reflections.

Students in New Zealand reported on their work with the Mauri and Pacific Island populations in Ora Tora clinics, which included addressing respiratory diseases, wound care and teaching diabetic patients' dietary advice to control unstable blood sugars.

Many students reflected on the inequity of health care resource allocation:

"I was frustrated and saddened to see how the system works here for the poorest populations". (CR)

"Most of the poor families could not afford many of the drugs, scans, X-rays and MRIs which is a major barrier to providing good health care". (CR)

"During my first week I was feeling overwhelmed by the poverty I was seeing in the streets and in the hospital. I did not think there would be children sleeping in cardboard boxes in the streets". (CR)

It was clear that all nursing students in both host countries felt that teaching at risk populations was a highlight of their trip (CRs) and there was an undercurrent of profound student learning through this teaching process (CRs, 
FGDs). Pre-departure orientation focused on teaching students important elements of vector control strategies (i.e., daytime biting mosquito, importance of wearing long sleeves and importance of diminishing stagnant water sources to decrease risk of dengue) and how to teach mothers to make their own oral rehydration solution (WHO 2007) to tackle childhood dehydration and diarrheal disease, which students implemented on the pediatric and dengue units in hospital and with urban and rural scavenger families in the community.

"One of the profound experiences I had was with a young mum down in ER (with her baby). She was dealing with diarrheal disease and we had learned the recipe for oral rehydration solution. She spoke really good English. I said I'm going to teach you how to prevent this in the future, and this is how you stop it, and I want you to go home and teach all your friends- everyone you know, you can do good in the world". (FGD)

Prior to their many teaching sessions students tried, with the help of a local translator, to determine existing knowledge levels of the families, and were surprised to discover that in some very rural Filipino communities cultural beliefs exist associating witchcraft with Dengue fever. Many students perceived health promotion to impoverished populations as more critical due to the urgency of preventing imminent mortality versus distant morbidity: For example, helping local communities understand principles of vector control to prevent Hemorrhagic Dengue Fever was viewed as more vital than teaching Canadian school children smart drink choices to prevent diabetes in later life.

The nursing students working in the Philippines described caring for patients with dengue fever, Tuberculosis, community-acquired pneumonia and pediatric diarrheal disease and recognized these as important infectious diseases affecting vulnerable populations. Additionally, they described challenges influencing the chain of disease transmission for example: lack of running water, soap, and medical supplies.

An interesting study finding was that $100 \%$ of students rated their cultural competency as "excellent" upon return to Canada. They rated their critical thinking skills, advocacy skills and relational practice skills as "very good". They were less generous rating their public health knowledge. Many felt they had not had enough opportunity to practise psychomotor skills during their overseas placement, resulting in a "fair" rating. Figure 3 outlines students' self-rated competencies. 
Figure 3. Student Survey Results

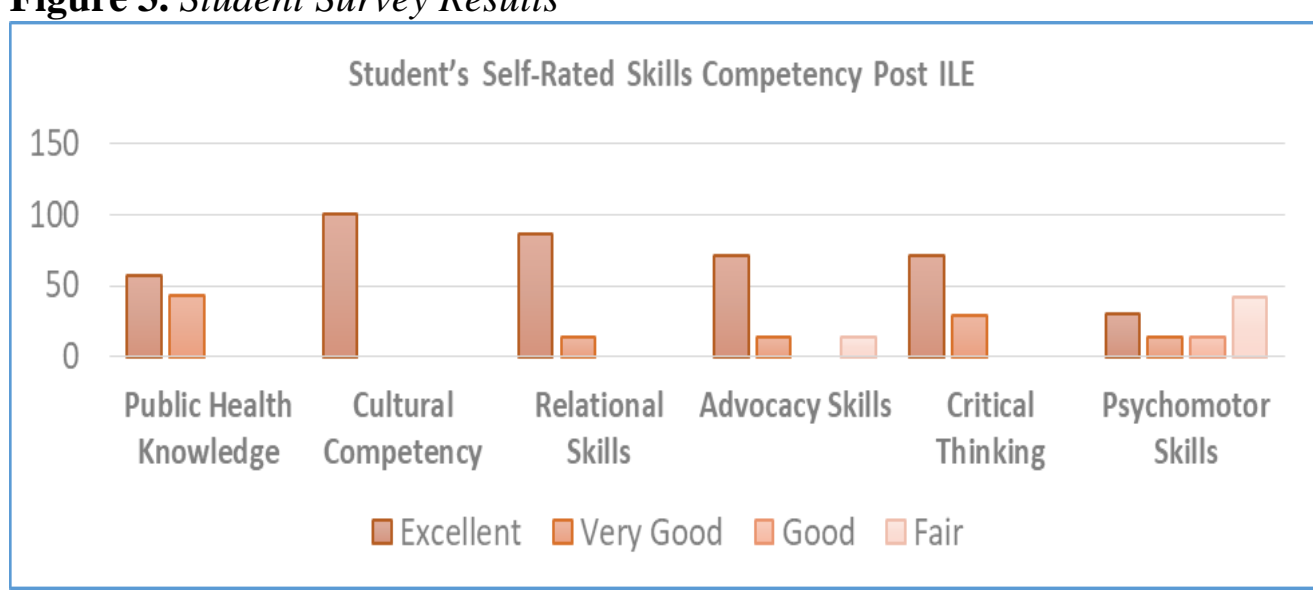

The Canadian students, many of whom came from affluent areas, found witnessing abject poverty uncomfortable and overwhelming. Despite observing these tough circumstances, which included the extreme lack of life saving resources, all students expressed gratitude for this life-changing opportunity by the end of their intense placement.

"I felt fortunate to participate in global nursing to expand my nursing knowledge of health ethics". (CR)

"I felt very inspired by the scavenger community experience-seeing what makes a sustainable healthy community!". (CR)

"This was an intense learning experience that challenged me both as a person and as a nursing student. It enhanced my empathic nature, gave me curiosity to learn about global health issues, improved my communication skills and helped me grow physically, emotionally and ethically as an individual. I loved every minute of it!". (CR)

\section{Objective 4: Student Personal and Professional Growth}

Overall, and notwithstanding the challenges they encountered, all students reported tremendous personal and professional growth during this international experience. Data from FGDs and CRs, coupled with researcher observation, highlighted how the ILE prompted a juncture that could be seen as the beginning of a multifaceted and individual journey towards cultural competence. These findings are congruent with previous research (e.g., DeLong 2015, Montenery et al. 2013, Button et al. 2005).

Attributes central to the concept of cultural competency are: cultural awareness; cultural sensitivity; cultural knowledge and cultural skills (Cai 2016). These attributes provide a frame for examining students' personal and professional growth over the course of the ILE and will be explored further in the discussion. 


\section{Cultural awareness}

Nursing students are exposed, through multiple formal and informal learning processes over the course of nursing school, to the concept of cultural awareness; that is, the recognition that different cultural attributes influence health (CNA 2018). Prior to their departure, the nursing students were confident in their awareness of cultural diversity and their cultural competence in general (FGD). Certainly, they had already been presented with multiple opportunities in the curriculum to experience different cultures that extended beyond definitions limited to ethnicity (Blanchet Garneau and Pepin 2015); however, the ILE provided incredible depth to the learning they had acquired thus far.

"It has been very important for me to always take a step back and try to understand some of the cultural values before judging actions that I may normally consider confusing". (CR)

"This was such a huge opportunity to understand how social determinants of health impact families through culture". (CR)

"There is a language barrier but body language and tone speak volumes". (CR)

\section{Cultural sensitivity}

Cultural sensitivity is an "appreciation, respect and comfort" for and with the cultural diversity of patients and clients (Cai 2016). Building on their emerging sense of cultural awareness, the students began to recognize their own cultural values and became more sensitive to the multiple perspectives of what healthcare is and means. For the majority of the students, this was unexpected and akin to what Jenkins et al. (2011) describe as "nursing shock". While the impact of the initial jarring exposure did seem to dissipate, as Jenkins et al. also reflect, the experience seemed to be more of a process of being "sensitized" to alternate interpretations of taken-for-granted ideals in a Canadian context and understanding that those customs are not necessarily the norm or even available elsewhere.

"My initial feelings were disbelief and shock!" [that things were not done as in Canada]. (CR)

"This young man (with a blunt trauma head injury) needed a CT scan and was not receiving it due to his family having to allocate funds to pay-they were [the equivalent of seven Canadian dollars] short...it is likely this young man died due to his injuries...". (CR)

Building on cultural awareness, cultural sensitivity refers to the idea that "both appreciation and respect are based on feeling comfortable within cultural diversity" (Cai 2016: 270). Understandably, there were differences in the degree of initial sensitization experienced by students from the Philippines versus New Zealand, where the hospital setting did not differ substantially from a Canadian setting. Many students in the Philippines talked about the importance of "getting comfortable with the uncomfortable" (FGD), which can 
be seen as a critical step in cultural sensitivity. (FGD) Many examples in the final CR demonstrated less judgment of local belief systems and more acceptance of the differences they observed in patient care.

"Being able to work in the Ora Toa clinic (New Zealand) has shown me that in order to improve the health of patients in the community, more preventative health care must be provided, rather than treating diseases when they occur!". (CR)

"One of the biggest learning outcomes for me is that I will never be protected from culture shock.... and that open-mindedness and respect is not equivalent to intercultural competence". (CR)

\section{Cultural knowledge}

Cultural knowledge is described by Cai (2016) as striving for a comprehensive educational foundation regarding a particular culture. Students recognize this in terms of the unique culture of their particular field school but also in terms of the culture of nursing within the overarching culture.

"It has become apparent to me that we may have things to teach each other as nurses". (CR)

"It is important to realize that resource allocation is not always better in Canada, [we] can be towards the other end of the spectrum with overuse and misuse of services". (CR)

Cultural skills, or the practise of incorporating culturally relevant assessments into nursing care (Cai 2016), seemed to be highlighted in the students' discussions and reflections of the varied and diverse issues they brought forward as ethical challenges.

"It has been ethically challenging at times [in the Philippines]. I realize I am a visitor to the country and I found it conflicting to understand where that boundary may lie: being respectful of culture and traditional ways of caring for Filipino patients...". (CR)

"In class, we learned about the SDOH and the role they play in health of an individual but this real-life experience put those factors into perspective". (CR)

"While the baby was in intensive care unit, the mom just left him there, neglected...the inequities of the system made me think that she might not be able to care for all her children, or even one, because of her economic status and complete lack of resources". (FGD)

Students realized the importance of cultural skills in practice, that their scope of nursing involved far more than merely performing set tasks.

"I realize more than ever that the care of a nurse is far more than just task oriented; it is about connecting with your patient and being fully present". (CR) 
"I realize there must be a balance between advocating for the patient in a respectful manner and stepping aside, but at this point in my training I feel that I have not fully grasped this yet". (CR)

In particular, as the students worked through ethical considerations, they came to realize that recognizing contradictions can be seen as an important aspect of self-reflection of their own cultural competence.

"I have not been talking about these experiences with others, not sure how much I could tell them-my family wondering how could it all be negative? My travel afterwards was talked about more...". (CR)

"I felt guilt coming from [a developed] country...and I did not want the patient...to feel I was judging them on the way they lived". (CR)

"This was harsh but what I learned here can't be learned anywhere else and I will use this experience in positive ways". (FGD)

All respondents echoed this sentiment:

"The trip did an amazing job of not only solidifying our knowledge but also pushing us out of our boundaries and challenging our beliefs". (FS)

\section{Discussion}

\section{Host Countries' Perceptions "Canadian Students Have Heart"}

Collaboration with the host country nursing staff was a critical, yet surprisingly often overlooked, research consideration. Kokko (2011) conducted a systematic literature review of seven empirical studies describing nursing students' studies abroad with a focus on cultural competence, yet none of these studies included the perceptions of their hosts. However, global host partner perspectives were the focus of one well designed qualitative study in the Dominican Republic (Underwood et al. 2016: 357) where engagement and collaboration of the host institutes provided a well needed "platform from which to intentionally dialogue with host partners in order to gain their context specific insights". The importance of this shared insight cannot be overstated. Host staff's perceptions stemming from this research helped to establish a more equal partnership. Although most staff in both countries surveyed were very pleased with the Canadian nursing students' compassion, flexibility and desire to help, local staff were able to provide insight into the divergent scope of nursing practice between countries, the importance of a more profound cultural orientation to the high intensity areas, and the need for these exchanges to be longer for more meaningful learning to occur. Kokko asserts that for the development process required to gain true cultural competence, longer exchanges are necessary. This is supported by other researchers whose analysis of the length of exchanges ranged from eight days to 21 days total (Browne and Fetherston 2018). This five-week study 
exceeded these shorter exchanges considerably yet host country staff rightly felt it was still an insufficient length of time for the students to understand the rich complex culture and therefore limited their ability to experience a profound cultural shift.

There is the perception that the interconnections made with host countries with a North American Health Care programme can have ongoing benefits. One can argue that globalization and the interrelationship of local and global contexts can create beneficial opportunities for students and their teachers to contribute to their hosts in international placements. Although further research is needed to critically examine contributions made to the host countries, the desire to contribute and altruism remain strong motivating factors (Gower et al. 2016, Burgess et al. 2014, Kumwenda et al. 2014, ReimerKirkham et al. 2009, Wehbi 2009).

If study abroad programmes are developed with effort and expense, to build alliances and partnerships across cultures with their host countries, the financial burden for students should also be addressed to allow greater access to study abroad programmes. Given that inadequate funds can be a primary deterrent for many students, one initiative may include implementing a program that offers greater access to funding specifically to support students considering study abroad including scholarships, bursaries or loans with generous terms of repayment.

\section{Students' Motivating Factors "I Went for the Opportunity to Travel-Experience Culture Shock!"}

While ILEs can create valuable learning opportunities for nursing students studying abroad, there is a danger that students choose to pursue these culturally diverse nursing placements for their "exotic" and "adventurous" elements rather than the pursuit of global health knowledge, professional growth and cultural competence. The motivation to study abroad for some students seemed disconnected from the stated learning outcomes for the course. As identified in other studies "cultural tourism" can be a dangerous motivating factor (Ramsden as cited in Racine and Perron 2012). This complex balance between providing a culturally rich and safe environment for young (often inexperienced) students, versus the opportunity of an adventure tour and the real potential for helicopter epidemiology (Jamrozik 2006) proves the key to the overall success of ILEs.

Students' responses concurred with literature regarding personal safety fears, in addition to the stressors they experienced through exposure to wideranging cultural differences (Kent-Wilkinson et al. 2015, Kelleher et al. 2016, Morgan 2012, Ruddock and DeSalles Turner 2007, Tyagi et al. 2006).

While such international experiences can create valuable learning opportunities, students also report concerns about the physical and emotional challenges (Gower et al. 2016, Tyagi et al. 2006). Our results concur with these findings where students voiced a tangible need for better preparation to work in resource poor settings, and the importance of personal resilience during the ILE (FGD). Lack of supplies, lack of cleanliness, inability to perform hand hygiene 
were listed among physical limitations. This finding was not unique to our study (Gower et al. 2016, Racine and Perron 2012, Tyagi et al. 2006).

A second issue worthy of consideration is the impact that student participants exert on their peers upon return, and the influence on nursing programs in general as they may ultimately draw upon their cultural journey and overseas nursing experience. Indeed, one can argue that these participants will potentially influence others long after they graduate and contribute positively to their local health care system to increase awareness and improve the cultural sensitivity in their profession. The underlying notion that those study abroad participants can be better positioned to shape their practice with a sense of cultural safety and a higher degree of cultural competence is an area that requires further study.

Recognizing the inherent value to the individual student participant and to the overall quality of health care programs, one also needs to reflect on the challenges that may deter future participants or derail future ILEs. In the case of Camosun College, the initiatives to mount a study abroad program in both 2017 and 2018 were cancelled due to the insufficient enrollment, and difficulty recruiting educators with pertinent global health experience. Given the identified deterrents such as inadequate funds and the impact on students' and faculty's family and work responsibilities, the question of how healthcare departments can better support interested parties is raised. Effective promotion of study abroad opportunities must not only address the motivating factors, but the important deterrents need to be considered in a thoughtful and clear fashion, both for students and faculty members.

\section{Global Health Knowledge: "Poverty is our Biggest Enemy"}

Many students are motivated to study abroad for a multitude of reasons as previously discussed in this paper. One criticism of ILEs described by our research and by other studies (Racine and Perron 2012) is that if not managed professionally with a rich cultural and global health lens, overseas placements run the risk of becoming an exciting form of cultural tourism, and may therefore miss vital, unique and rich learning opportunities. The inclusion of an adapted version of Riner's (2011) framework into this study design coupled with other important global health concepts (including tropical diseases, $\mathrm{SDOH}$, and health promotion) helped us gain a richer understanding of the value of these ILEs from a global health perspective.

The importance of the students' CRs as a valuable research tool is evident and this was found equally important in other studies (Ruddock and DeSalles Turner 2007). It was often after challenging shifts that students were able to explore their beliefs, values, judgments and underlying knowledge around complex patient care issues through these intense journal entries that served as important data sources but more importantly helped students process some of their difficult nursing experiences. Many of the core SDOH which they admitted to not adequately considering in Canada became apparent as they confronted abject poverty, lack of hospital supplies, and poorly educated families with sick children. 
Students' self rated competencies of their global health knowledge and cultural competency showed an improvement following their international placement. Interesting to note however that their very high self rating of cultural competency often did not align with the researcher's personal observations. At times a few students appeared judgmental and held an air of superiority. Racine and Perron (2012) explore similar findings. The true depth of this learning may not become apparent until a later time in the students' careers as they gain maturity, experience and better insight into their experience. It would be important to survey the students several years after their ILE, something our study did not consider, but has been highlighted by research done by Kokko (2011: 681) where "nurturing after graduation" was recommended to sustain cultural growth.

There was an undercurrent of intense student learning through their teaching process. Teaching at risk populations was a highlight in the majority of students' CRs (25/32). Although patient education is an important part of their Canadian nursing curricula and learning outcomes, students seemed to place more value on this role and expressed pride in this element of their nursing practice. It was evident that students' knowledge of infectious diseases and the impact of the SDOH was enhanced through a combination of taught lectures by Canadian instructors prior to and during the practicum, through their weekly critical reflections, and by the complexity of patients experiencing a multitude of infectious diseases students do not often witness in their routine Canadian nursing training. For example, students' recognition that early detection of the signs and symptoms of dengue fever and subsequent prompt treatment has the potential to reduce patients' case fatality rates to less than one percent (WHO 2019) was transformative.

Students' Personal and Professional Growth: "Getting Comfortable with the Uncomfortable"

Participating in an ILE has a profound impact on the professional and personal growth of students (e.g., Button et al. 2005, Lee 2004). Our results demonstrate that such growth can be framed within the concept of cultural competence, which can also be viewed as a gradual "developed capacity of nurses to provide safe and quality healthcare to clients with different cultural backgrounds" (Cai 2016). That is, cultural competence is a continuous lifelong dynamic process requiring an emphasis on perpetual development and explicit focus of cultural awareness, cultural sensitivity, cultural knowledge, and cultural skills (Cai 2016). As cultural competence is considered a requisite entry-to-practice ability for Canadian nurses (CNA 2018), the notion of supporting cultural competence in nursing students and novice nurses requires further attention. This is no small task as it necessitates that we "become comfortable with the uncomfortable".

Cultural competence, particularly cultural skills, were highlighted in the student nurses' discussions regarding ethical challenges. Benner et al. (2010) argue that nursing ethics has consistently been taught well across nursing education programs. Further theorizing of ethical comportment has proved a 
useful construct in this area (Day and Benner 2002, Benner et al. 2008). Ethical comportment is the ability to demonstrate "appropriate use of knowledge, skills of care and relations, and communication with patients and colleagues" (Benner et al. 2010). Extending this definition, cultural comportment can be seen as an educational opportunity at the nexus of nursing ethics and cultural competence.

The challenge is not only to create opportunities along this journey for nursing students (and faculty) to explicitly focus on the dynamic nature of cultural competence, but to also integrate the central attributes outlined above into everyday practice. Viewing cultural comportment as an avenue to support and role model cultural competence may provide nursing students (and faculty) with the confidence necessary to sustain and embed this process in a way that will inform their nursing practice across an entire career. Including cultural competence within ethics discussions, and further supplementing those discussions within the context of ethical comportment, will assist the promotion of a life-long curiosity in this regard. ILEs can be seen as a disruptive pedagogical technique that will focus nursing students on the importance and value of cultural competence, and cultural comportment, both abroad and at home.

\section{Limitations}

The primary sampling technique was based on convenience and thus, the group consisted of students who were financially able to participate. In this way, the group may not be representative of overall nursing student population. As well, the data was collected over a short time frame, providing a snapshot of the ILEs offered at that time. International placement sites will vary across time, depending on student interest and faculty experience and availability. The snapshot of the ILEs examined only considered short term learning outcomes; a longitudinal study is recommended to examine sustained impact for students as often the most important learning is not actualized immediately upon return. There are also concerns that the host country nurses may only provide positive feedback with little critique via the questionnaires due to cultural etiquette, language barriers and a desire not to offend their guests.

\section{Conclusion}

In summary, the study findings identify that host country staff benefited significantly from collaborating with our ILE students and it was important to include their opinions into the study design. The Canadian student participants developed a deeper awareness of the host culture coupled with a better understanding of the key SDOH in both settings. The students revealed exponential growth both personally and professionally as they embarked on a challenging journey on the road to cultural competence and cultural comportment. This paper puts forth new suggestions for future ILE practices and areas worthy of further exploration. It is hoped that findings from this 
research will inform the global debate not only on the value and importance of ILEs, but on ways to ensure cultural competence is embedded into nursing practise from graduation with a sustained and richer global health focus.

\section{Recommendations}

- Apply a post colonial lens when constructing learning activities for nursing students attending ILEs with the intention of supporting the inclusion of host country perspectives.

- Prepare students and faculty intentionally, with a focus on the importance of an in-depth cultural workshop from a health perspective with clearer objectives that relate to the $\mathrm{SDOH}$, tropical medicine, cultural competence and cultural comportment - designed explicitly to move beyond cultural tourism.

- Develop a comprehensive orientation and evaluation toolkit for both instructors and students, which includes a clear global health framework with related learning outcomes.

- Ensure a formative evaluation and debriefing across the entire ILE, addressing immediate concerns (including post traumatic distress) with follow up upon return to Canada.

- Embed explicit nursing ethics discussions and frameworks to consolidate ethical dimensions of practice, which support the acquisition of cultural competency.

- Assess impact of the intentional critical reflections on students' journey to cultural competence through the lens of personal and professional growth.

- Develop a comprehensive psychomotor skills-workshop upon returning from a low-income country is warranted.

- Provide simulated learning experiences addressing both assessment skills and global health concepts to prepare no only students attending ILEs, but those who participated in domestic placements.

- Examine the long-term impact of ILEs on students' cultural competence journey post-graduation.

\section{Acknowledgements}

Our thanks to both host country staff who graciously welcomed us into their hospitals and participated in this research, to the student participants for their enthusiasm, compassion and hard work, to our partners Cathy McDonald and Marlene Welsh, and finally to Camosun College who funded our flights, accommodation, and the development/research time to make this study and dissemination of these results possible. 


\section{References}

Benner P, Sutphen M, Leonard-Kahn V, Day L (2008) Formation and everyday ethical comportment. American Journal of Critical Care 17(5): 473-476.

Benner P, Sutphen M, Leonard V, Day L (2010) Educating nurses: A call for radical transformation. San Francisco, CA: Jossey Bass.

Blanchet Garneau A, Pepin J (2015) Cultural Competence: A Constructivist Definition. Journal of Transcultural Nursing 26(1): 9-15.

Bohman DM, Borglin G (2014) Student exchange for nursing students: does it raise cultural awareness? A descriptive, qualitative study. Nurse Education in Practice 14(3): 259-264.

Browne C, Fetherston C (2018) How do we facilitate international clinical placements for nursing students: a cross-sectional exploration of the structure, aims and objectives of placements? Nurse Education Today 66: 1-7.

Brown M, Appiah Goateng E, Evans C (2016) Should I stay or should I go? As systematic review of factors that influence healthcare students' decision around study abroad programmes. Nurse Education Today 39: 63-71.

Burgess CA, Reimer-Kirkham S, Astle B (2014) Motivation and international clinical placements: Shifting nursing students to a global citizenship perspective. International Journal of Nursing Education Scholarship 11(1): 1-8.

Button L, Green B, Tengnah C, Johansson I, Baker C (2005) The Impact of International Placements on Nurses' Personal and Professional Lives: Literature Review. Journal of Advanced Nursing 50(3): 315-324.

Cai D (2016) A concept analysis of cultural competence. International Journal of Nursing Sciences 3(2016): 268-273.

Campesino M (2002) Commentary: problematic issues in cultural comparisons. Journal of Professional Nursing 18 (6): 343-345.

Campinha-Bacote J (2011) Coming to know cultural competence: An evolutionary process. International Journal for Human Caring 15(3): 42-48.

CAN-Canadian Nurses Association (2018) Position statement: Promoting cultural competence in nursing. Ottawa, Canada. Retrieved from: https://bit.ly/2J3dHtG. [Accessed: November 18, 2018]

Day L, Benner P (2002) Ethics, ethical comportment, and etiquette. American Journal of Critical Care 11(1): 76-79.

DeLong M (2015) Nursing Student International Experiences: An overview of the literature. Unpublished submission of course requirements for $\mathrm{N}$ 611, Trinity Western University.

Frisch NC (1990) An international nursing student exchange program: An educational experience that enhanced student cognitive development. Journal of Nursing Education 29(1): 10-12.

Government of Canada (2018) Social determinants of health and health inequalities. Retrieved from https://bit.ly/2Fa699p. [Accessed: December 1, 2018]

Gower S, Duggan R, Dantas J, Boldy D (2016) Motivations and Expectations of Undergraduate Nursing Students Undertaking International Clinical Placements. Journal of Nursing Education 55(9): 487-494.

Greatrex-White S (2007) Uncovering study abroad: foreignness and its relevance to nurse education and cultural competence. Nurse Education Today 28(5): 530-538.

Holmes D, Zayas L, Koyfman A (2012) Student objectives and learning experiences in a global health elective. Journal of Community Health 37(5): 927-934.

Jamrozik K (2006) The epidemiology of colonialism. The Lancet 368(9529): 4-6.

Jenkins E, Balneaves LG, Lust A (2011) Students and international nursing shock. The Canadian Nurse 107(7): 26-30. 
Kelleher S, Fitzgerald S, Hegarty J (2016) Factors that influence nursing and midwifery students' intentions to study abroad: A qualitative study using the theory of planned behaviour. Nurse Education Today 44: 157-164.

Kent-Wilkinson A, Dietrich Leurer M, Luimes J, Ferguson L, Murray L (2015) Studying abroad: exploring factors influencing nursing students' decision to apply for clinical placements in international settings. Nurse Education Today 35(8): 941-947.

Keogh J, Russel-Roberts E (2009) Exchange programs and student mobility: meeting student's expectations or an expensive holiday? Nurse Education Today 29(1): 108-116.

Kokko R (2011) Future nurses' cultural competencies: what are their learning experiences during exchange and studies abroad? A systematic literature review. Journal of Nursing Management 19(5): 673-682.

Kulbok PA, Mitchell EM, Glick DF, Greiner D (2012) International experiences in nursing education: A review of the literature. International Journal of Nursing Education Scholarship 9(1): 1-21.

Kumwenda B, Royan D, Ringsell P, Dowell J (2014) Western medical students' experiences on clinical electives in sub-Saharan Africa. Medical Education 48(6): 593-603.

Lee NJ (2004) The impact of international experience on student nurses' personal and professional development. International Nursing Review 51(2): 113-122.

Montenery SM, Jones AD, Perry N, Ross D, Zoucha R (2013) Cultural competence in nursing faculty: a journey, not a destination. Journal of Professional Nursing 29(6): e51-e57.

Morgan DA (2012) Student nurse perceptions of risk in relation to international placements: A phenomenological research study. Nurse Education Today 32(8): 949-960.

Owen C, Breheny P, Ingram R, Pfeifle W, Cain J, Ryan M (2013) Factors associated with pharmacy student interest in international study. American Journal of Pharmacy Education 77(3): 54.

Racine L, Perron A (2012) Unmasking the predicament of cultural voyeurism: a postcolonial analysis of international nursing placements. Nursing Inquiry 19(3): 190-201.

Reimer-Kirkham S, Van Hofwegen L, Pankratz D (2009) Keeping the vision: sustaining social consciousness with nursing students following international learning experiences. International Journal of Nursing Education Scholarship 6(1): 1-6.

Riner ME (2011) Globally engaged nursing education: An academic program framework. Nursing Outlook 59(6): 308-317.

Ruddock HC, DeSalles Turner S (2007) Developing cultural sensitivity: nursing students' experiences of a study abroad programme. Journal of Advanced Nursing 59(4): 361-369.

Statistics Canada (2011) National Household Survey. Immigration and ethnocultural diversity in Canada. Analytical document 99-010-X. Retrieved from https://bit.ly/ 2XQyUxf. [Accessed: December 1, 2018]

Thorne S (2016) Interpretive Description: Qualitative Research for Applied Practice, $2^{\text {nd }}$ Ed. New York: Routledge.

Torsvik M, Hedlund M (2008) Cultural encounters in reflective dialogue about nursing care: a qualitative study. Journal of Advanced Nursing 63(4): 389-396.

Tyagi S, Corbett S, Welfare M (2006) Safety on elective; a survey on safety adverse events during electives. Clinical Medicine 6(2): 154-156. 
Underwood M, Gleeson J, Konnert C, Wong K, Bautista V (2016) Global host partner perspectives utilizing a conceptual model to strengthen collaboration with host partners for international nursing student placements. Public Health Nursing 33(4): 351-359.

Wehbi S (2009) Deconstructing motivations: Challenging international social work placements. International Social Work 52(1): 48-59.

WHO-World Health Organization (2007) The World Health Report 2007. A safer future: Global public health security in the $21^{\text {st }}$ century. Geneva, Switzerland.

WHO-World Health Organization (2019) Dengue and sever dengue: Key facts. Retrieved from https://bit.ly/2CpkP0Y. [Accessed: June 22, 2019]

Zorn C, Ponick D, Peck S (1995) An Analysis of the Impact of Participation in an International Study Program on the Cognitive Development of Senior Baccalaureate Nursing Students. Journal of Nursing Education 34(2): 67-70. 